\title{
Source and risk factors of a cutaneous anthrax outbreak, Jiangsu, Eastern China, 2012
}

\author{
J. L. HU ${ }^{1} \dagger$, L. L. CUI ${ }^{2,3} \dagger$, C. J. BAO ${ }^{1 *}$, Z. M. TAN ${ }^{1}$, S. RUTHERFORD ${ }^{4}$, \\ L. YING ${ }^{5}$, M. L. ZHANG ${ }^{6}$ AND F. C. ZHU \\ ${ }^{1}$ Department of Acute Infectious Diseases Control and Prevention, Jiangsu Province Center for Disease Control \\ and Prevention, Nanjing, PR China \\ ${ }^{2}$ Jinan Municipal Center for Disease Control and Prevention, Jinan, PR China \\ ${ }^{3}$ Chinese Field Epidemiology Training Program, Beijing, PR China \\ ${ }^{4}$ Centre for Environment and Population Health, School of Environment, Griffith University, Brisbane, Australia \\ ${ }^{5}$ Lianyungang Municipal Center for Disease Control and Prevention, Lianyungang, PR China \\ ${ }^{6}$ Ganyu County Center for Disease Control and Prevention, Ganyu, PR China \\ ${ }^{7}$ Jiangsu Province Center for Disease Control and Prevention, Nanjing, PR China
}

Received 9 October 2015; Final revision 15 May 2016; Accepted 25 May 2016;

first published online 9 June 2016

\section{SUMMARY}

Anthrax is still a severe public health problem and threat to human health. A cutaneous anthrax outbreak occurred in Jiangsu Province, a non-endemic anthrax region of eastern China, from July to August 2012. Epidemiological and laboratory investigation were initiated to trace the source of infection and identify the risk factors of the outbreak. On 25 July 2012, 17 persons were exposed to a sick cow, which had been imported from northeast China a few days previously. Of the 17 exposed, eight developed symptoms between 1 and 8 days and were diagnosed as cutaneous anthrax cases. Three main genes of Bacillus anthracis were detected from both human and cow meat samples, indicating that the outbreak was associated with this infected cow. A retrospective cohort study showed that contact with blood and presence of skin damage contributed to the case infection with $B$. anthracis. The outbreak highlights the need to enhance quarantine for imported livestock, which should have been vaccinated prior to importation, the significance of education for high-risk individuals, and training for primary healthcare workers even in anthrax-free areas.

Key words: Cutaneous anthrax, imported, outbreak, retrospective cohort study, risk factor.

\section{INTRODUCTION}

Anthrax is an infectious zoonotic disease caused by Bacillus anthracis, a large, Gram-positive, aerobic, spore-forming bacillus. Human infections usually

\footnotetext{
* Author for correspondence: Dr C. J. Bao, Department of Acute Infectious Diseases Control and Prevention, Jiangsu Province Center for Disease Control and Prevention, No. 172, Jiangsu Road, Nanjing 210009, People's Republic of China. (Email: bao2000_cn@163.com)

$\dagger$ These authors contributed equally to this work.
}

result from contact with infected animals or contaminated products. Over 20000 human cases are estimated to occur worldwide every year [1], with most cases coming from low-income countries and areas [2]. Human cases with $B$. anthracis infection are not uncommon in developed counties of Europe and are seen occasionally in specific parts of the United States, such as eastern North Dakota, and western Texas in relation to sporadic livestock and wildlife outbreaks [3, 4]. Some of the cases are related to imported animal products like hides $[5,6]$ and recently 
(a)

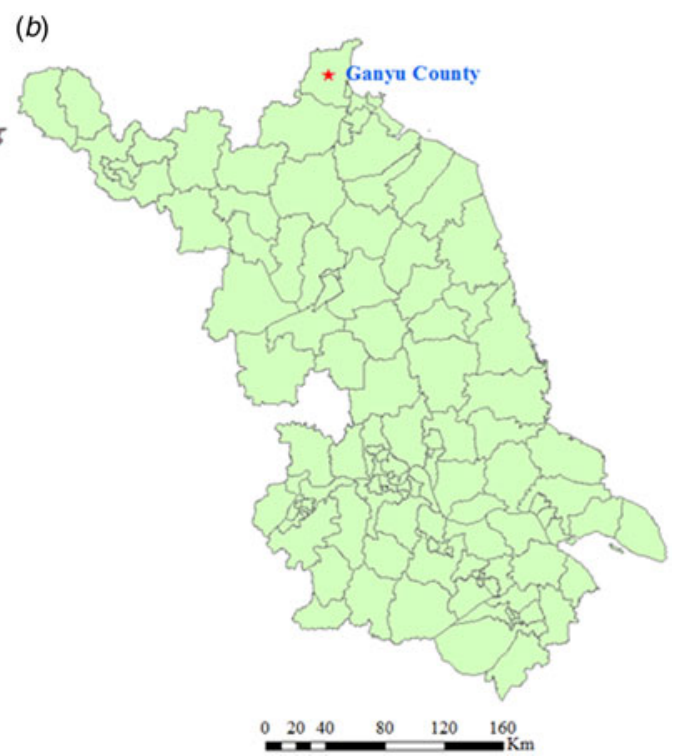

Fig. 1. Map showing the site of a cutaneous anthrax outbreak associated with an imported sick cow in Jiangsu Province in a non-endemic anthrax region, eastern China during July-August 2012. (a) Map of China with a red dot showing the possible source of the sick cow, in which the numbers of annual average reported human anthrax cases (reported through a national system for reporting of notifiable infectious diseases) are highlighted in different colours, and shows as one province in a non-endemic anthrax region. (b) Map of Jiangsu Province and the county where the cutaneous anthrax outbreak occurred during July-August 2012.

more cases have been ascribed to heroin in drug injection users in European countries [7]. In addition, the incidents associated with deliberate release that occurred in multiple states of the United States in 2001 forced consideration of the potential huge impact of using spores of $B$. anthracis as a biological weapon $[8,9]$. Thus, anthrax still imposes severe public health challenges for humans and animals [2, 10].

About 300 anthrax human cases have been reported in China annually in the past 5 years, with the majority originating from western animal husbandry regions (Fig. 1). Jiangsu Province, which is in the east of China, consists mainly of plains and a network of rivers, and an industrial region with some agricultural production. Jiangsu Province belongs to a non-endemic anthrax area, and infections with $B$. anthracis both in humans and animals are very rare. During the second half of the 20th century, there were only 12 human cutaneous anthrax cases reported in Jiangsu Province, and no cases have been reported since 1998 (Annual Report of epidemic in Jiangsu Province, unpublished data). However, from 25 July to 2 August 2012, an outbreak associated with an imported cow occurred in Ganyu County, Jiangsu Province (Fig. 1). The objective of this paper is to determine the dimensions of the outbreak, articulate the risk factors that contributed to the outbreak, and suggest monitoring, control and prevention strategies in anthrax endemic-free areas, such as Jiangsu Province.

\section{METHODS}

\section{Case definition}

A confirmed case was defined as a clinically compatible illness with one of the following laboratory evidence: detection of both $B$. anthracis special genes and toxin genes [chromosome-specific $(r p o \mathrm{~B})$, toxin (pagA), and capsule (cyaA) genes] by real-time polymerase chain reaction (PCR) from an affected tissue or site; or the presence of antibodies against $B$. anthra$c i s$ in sera through immunodiagnostic testing.

A probable case was defined as a clinically compatible illness with no laboratory evidence, but with exposure to infected sick or dead animals or their products, or epidemiologically linked to a confirmed case.

\section{Sample collection and laboratory testing}

Samples were obtained from seven human cases including five biopsy specimens and seven serum specimens; three frozen beef samples were collected from the slaughtered sick cow. Real-time PCR was used to detect the rpoB gene which is located in the 
bacterial chromosome and encoded $\beta$-subunit of the RNA polymerase, protective antigen gene (pag) which is located in plasmid pXO1, and capsular gene $(\operatorname{cap} \mathrm{A})$ which is located in plasmid $\mathrm{pXO}$. Meanwhile, general PCR was used to detect special toxic genes including the oedema factor gene (cya), the lethal factor gene (lef), and the protective antigen gene (pag) as well as the capsular gene (capA) related to spore formation [11]. Colloidal gold (Jinhao, China) was used to detect the anti-capsule antibodies. Culture of B. anthracis was not performed due to potential biosafety issues and also because all cases were treated with antibiotics before their blood specimens were collected.

\section{Risk factor investigation and statistical analysis}

A retrospective cohort study was used to clarify risk factors that contributed to the infection. All 17 individuals (eight cases, nine controls) consented to be participants. A standardized questionnaire developed by Jiangsu Province Centre for Diseases Control and Prevention was used to collect information such as specific exposure mode, presence of skin wounds, and of mosquito bites during and before slaughtering. The effect of different exposures was evaluated by the value of relative risk (RR) and $95 \%$ confidence interval (CI) estimated by the Mantel-Haenszel method. A CI range which did not include 1 was considered significant.

\section{Ethical approval}

An ethics waiver was granted and authorized according to the Chinese National Emergent Public Health Events Act. Investigation and data collection related to the outbreak were exempt from institutional board assessment.

\section{RESULTS}

\section{Epidemiological investigation and active case-finding}

The index case was notified to public health officers on 2 August 2012 by an alert physician in a tertiary infectious disease specialist hospital, who raised the diagnostic possibility of cutaneous anthrax when examination revealed typical skin lesions on the patient's little finger, i.e. vesicles with a central black eschar (Fig. 2). Before this, the index case had visited different types of hospital (local village, township, county) between 29 July and 1 August, and was

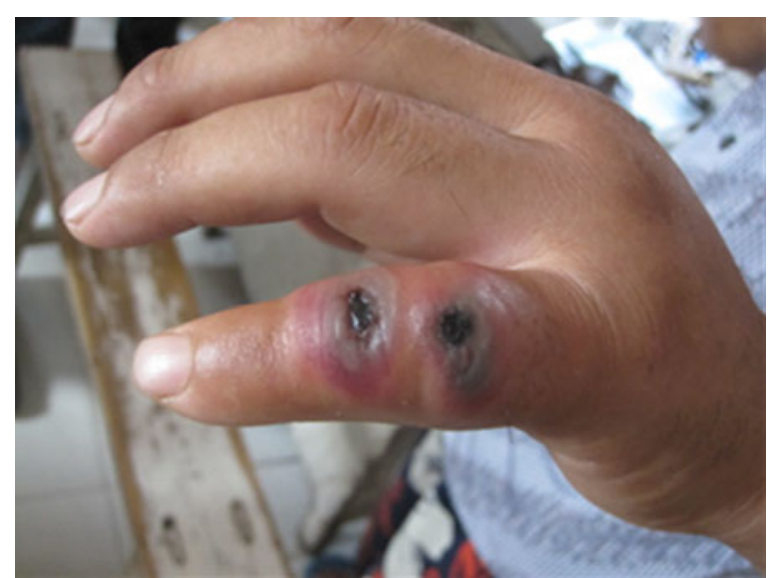

Fig. 2. Typical skin lesions (vesicles with central black eschar) on the little finger of the index case that developed several days after he slaughtered and butchered a sick cow on 25 July 2012.

treated with antibiotics and dexamethasone but no suspected diagnosis of $B$. anthracis infection had been made. The index case was a butcher who on 25 July slaughtered a sick cow with other persons without using any personal protective equipment. His little finger was cut by a bone of the cow during the slaughtering process. The cow, purchased from a county in northeast China, became sick just after arriving in Ganyu County (Fig. 1).

After talking with the index patient, public health officials learned that a similar situation could have occurred in other persons who had participated in the slaughtering and butchering of the sick cow. Therefore, in order to detect potential cases of infection and determine risk factors contributing to infection, an active case-finding surveillance was initiated. The index case was interrogated in detail about which persons had participated in the slaughtering and butchering, who were the buyers of the sick cow products, and who were in charge of dealing with the hide and bones. A number of other steps were also taken. First, all individuals who had participated in the slaughtering or had contact with products such as skin, meat, organs, or blood were listed and traced. Fifteen persons were identified of whom six were confirmed as case-patients. Second, knowledge relating to clinical features of anthrax was widely publicized by media and via flyers. Third, local hospitals were alerted to report any cases with skin lesions, especially those who had a history of contact with sick or dead cattle before illness onset. One probable casepatient who picked up and ate a piece of discarded meat/organ was identified. 


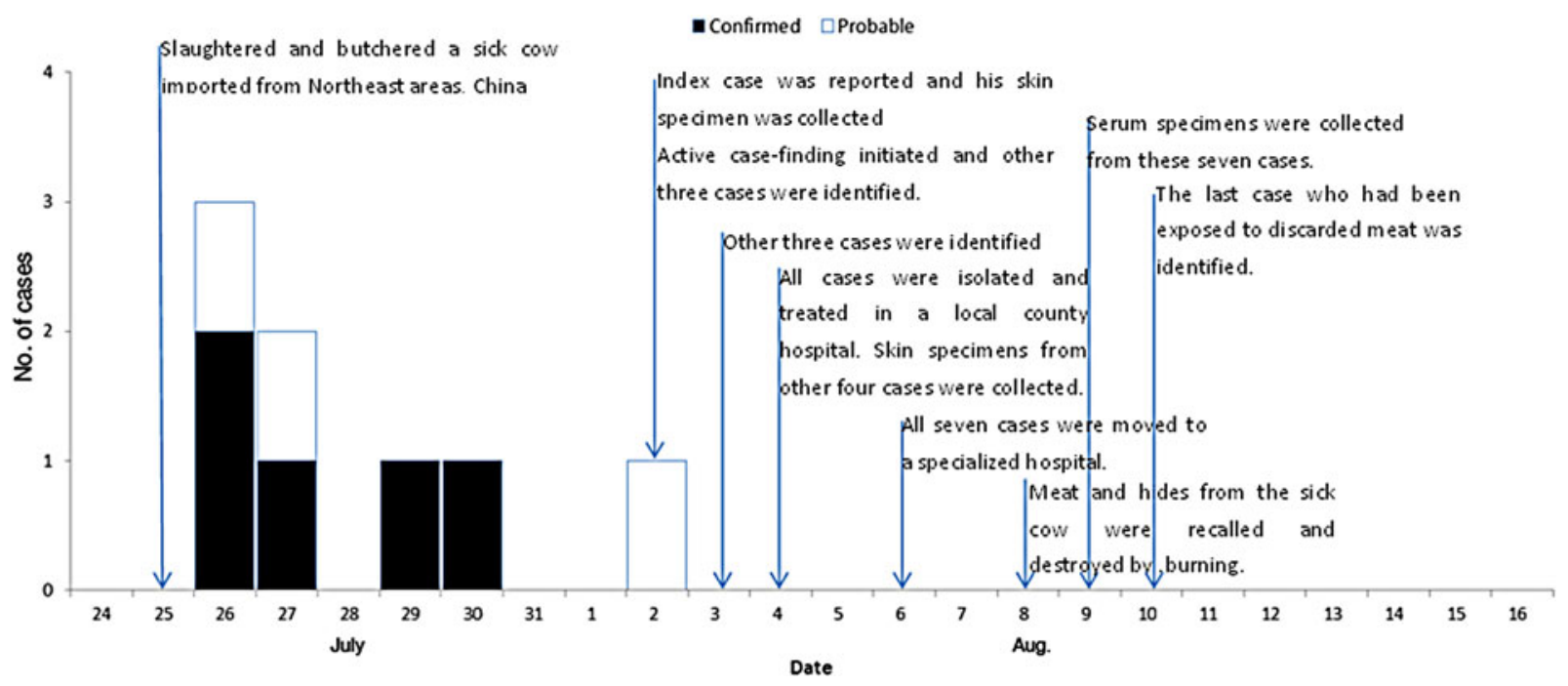

Fig. 3. Timelines and epidemic curve of eight cutaneous anthrax cases associated with an imported sick cow in a non-endemic anthrax region, eastern China, during July-August 2012. With the exception of the eighth case who was not hospitalized due to mild symptoms, all the other seven cases in the outbreak recovered and were discharged from hospital during 16-31 August.

\section{Demographic and clinical characteristics of cases}

With the exception of the index case, a total of 16 persons were identified who had been exposed to the sick cow through active case-finding. Of these, eight developed symptoms following exposure. Five of them were subsequently diagnosed as laboratory-confirmed cases and three as probable cases. The timelines and characteristics of the cases are given in Figure 3 and Table 1, respectively. The attack rate related to this outbreak was about $47 \%(8 / 17)$. All cases were classified as cutaneous anthrax. Five cases were butchers, two were housewives and one was a farmer. The average age of the six male and two female cases was 34 years (range $21-48$ years). The average number of skin lesions for each case was 2.75 (range 1-9). No persons died. With the exception of the eighth case who was not hospitalized due to mild symptoms, all other seven cases in the outbreak recovered and were discharged from hospital during 16-31 August. The pathways of exposure included slaughtering (five cases) and washing products (three cases). The median incubation period was 2 days (range 1-8 days).

\section{Laboratory investigation}

Biopsy specimens were obtained from $5 / 8$ cases and three samples from the sick cow. In four of the five cases and all three samples from the sick cow, chromosome-specific (rpoB), toxin (pagA), and capsule $($ cya $\mathrm{A})$ genes against $B$. anthracis were detected. Further molecular typing revealed that the genotype of the pagA gene, amplified from samples of four patients and of beef meat, respectively, was identical. Serum specimens were obtained from $7 / 8$ cases. In two of these cases anti-capsule antibodies against $B$. anthracis were detected. The above-mentioned three special genes and anti-capsule antibodies were detected from biopsy and serum specimens collected from the same case. Therefore, five of the eight cases were considered as confirmed cases and the other three were probable cases (Table 1).

\section{Risk factor analysis}

A retrospective cohort study was conducted to clarify risk factors which contributed to the infection with $B$. anthracis. All the 17 individuals who were exposed to the sick cow or its products were enrolled in the study. The factors included method of slaughtering, type of contacted products, with or without skin wound, and mosquito bites. Analysis showed that only two factors: contact with blood from the sick cow and presence of skin damage before exposure, contributed significantly to the infection. The RR values for these two risk factors were $2 \cdot 5(95 \% \mathrm{CI} 1 \cdot 3-4 \cdot 6)$ and $2 \cdot 8$ (95\% CI $1 \cdot 4-5 \cdot 7)$, respectively (Table 2 ).

\section{Control measures}

Some control and prevention measures were taken once the outbreak was detected. First, the local 
Table 1. Characteristics of eight cutaneous anthrax cases associated with an imported sick cow in Jiangsu Province, eastern China, July-August 2012

\begin{tabular}{|c|c|c|c|c|c|c|c|c|}
\hline Characteristics & Patient 1 & Patient 2 & Patient 3 & Patient 4 & Patient 5 & Patient 6 & Patient 7 & Patient 8 \\
\hline \multicolumn{9}{|l|}{ General information } \\
\hline Age (years) & 48 & 23 & 28 & 21 & 26 & 43 & 27 & 59 \\
\hline Sex & $\mathrm{M}$ & $\mathrm{M}$ & M & $\mathrm{M}$ & $\mathrm{M}$ & $\mathrm{F}$ & $\mathrm{F}$ & $\mathrm{M}$ \\
\hline Occupation & Butcher & Butcher & Butcher & Butcher & Butcher & Housewife & Housewife & Farmer \\
\hline Date of illness onset & 26 July & 26 July & 30 July & 27 July & 29 July & 2 August & 26 July & 27 July \\
\hline $\begin{array}{l}\text { Epidemiologically } \\
\text { linked to a sick cow or } \\
\text { its products }\end{array}$ & Yes & Yes & Yes & Yes & Yes & Yes & Yes & Yes \\
\hline $\begin{array}{l}\text { Incubation period } \\
\text { (days) }\end{array}$ & 1 & 1 & 5 & 2 & 4 & 8 & 1 & 2 \\
\hline Fever & Yes & Yes & Yes & No & No & No & Yes & No \\
\hline $\begin{array}{l}\text { Skin lesion such as } \\
\text { ulcers, papules, } \\
\text { erythema, vesicles, or } \\
\text { central black eschars }\end{array}$ & Yes & Yes & Yes & Yes & Yes & Yes & Yes & Yes \\
\hline $\begin{array}{l}\text { Location of skin } \\
\text { lesions }\end{array}$ & $\begin{array}{l}\text { Back of } \\
\text { hand and } \\
\text { little } \\
\text { finger }\end{array}$ & $\begin{array}{l}\text { Face, arm } \\
\text { and leg }\end{array}$ & Hand & Foot & $\begin{array}{l}\text { Arm and } \\
\text { leg }\end{array}$ & Thigh & Finger & Calf \\
\hline Number of skin lesions & 3 & 9 & 1 & 1 & 4 & 2 & 1 & 1 \\
\hline Isolation in hospital & Yes & Yes & Yes & Yes & Yes & Yes & Yes & No \\
\hline Survived & Yes & Yes & Yes & Yes & Yes & Yes & Yes & Yes \\
\hline \multicolumn{9}{|l|}{ Laboratory testing } \\
\hline Real-time RT-PCR & Positive & Positive & Positive & Positive & Negative & n.t. & n.t. & n.t. \\
\hline $\begin{array}{l}\text { Anti-protective } \\
\text { antigen } \mathrm{IgG}^{*}\end{array}$ & Negative & Negative & Positive & Negative & Positive & Negative & Negative & n.t. \\
\hline
\end{tabular}

n.t., Not tested.

* All seven serum specimens were collected on 9 August 2012.

Table 2. Risk-factor analysis of a cutaneous anthrax outbreak associated with an imported sick cow in Jiangsu Province, eastern China, July-August 2012

\begin{tabular}{|c|c|c|c|c|c|}
\hline \multirow[b]{2}{*}{ Type of exposure } & \multicolumn{2}{|c|}{ Exposed } & \multicolumn{2}{|c|}{ Non-exposed } & \multirow[b]{2}{*}{$\mathrm{RR}(95 \% \mathrm{CI})$} \\
\hline & Case & Control & Case & Control & \\
\hline Slaughtering & 7 & 3 & 1 & 6 & $4.9(0 \cdot 76-31)$ \\
\hline Deboning & 5 & 2 & 3 & 7 & $2 \cdot 4(0 \cdot 83-6 \cdot 8)$ \\
\hline Bloodletting, peeling, eviscerating, or washing & 2 & 1 & 6 & 8 & $1 \cdot 6(0 \cdot 57-4 \cdot 2)$ \\
\hline Eating meat & 1 & 1 & 7 & 8 & $1 \cdot 1(0 \cdot 24-4 \cdot 7)$ \\
\hline \multicolumn{6}{|l|}{ Contact with } \\
\hline Blood & 2 & 0 & 6 & 9 & $2 \cdot 5(1 \cdot 3-4 \cdot 6)$ \\
\hline Organs & 4 & 1 & 4 & 8 & $2 \cdot 4(0 \cdot 96-6 \cdot 0)$ \\
\hline Skin & 3 & 1 & 5 & 8 & $2 \cdot 0(0 \cdot 80-4 \cdot 8)$ \\
\hline Meat & 6 & 5 & 2 & 4 & $1 \cdot 6(0 \cdot 47-5 \cdot 7)$ \\
\hline Skin cut, lesion or bite by mosquitos before exposure & 3 & 0 & 5 & 9 & $2 \cdot 8(1 \cdot 4-5 \cdot 7)$ \\
\hline
\end{tabular}

RR, Relative risk; CI, confidence interval. 
veterinary department was informed about the outbreak. Second, all products linked to the sick cow were recalled and destroyed by burning. Third, all persons who were possibly exposed to sick or dead cattle were traced, given antibiotics as post-exposure prophylaxis for 3 days, and then followed for 12 days. Fourth, fact sheets and a newsletter were developed and distributed to both butchers and their families and local medical staff. Apart from including basic knowledge relating to anthrax, the ban on illegal slaughtering was especially highlighted.

\section{DISCUSSION}

Epidemiological, clinical and laboratory findings indicated that the outbreak which occurred in Ganyu County, Jiangsu Province from July to August 2012 was caused by $B$. anthracis. Slaughtering and butchering products of an anthrax-infected cow were found to be responsible for this outbreak. A retrospective cohort study indicated that both contact with blood of the sick cow and presence of skin damage before exposure were the two primary risk factors that favoured developing anthrax [12]. The infection source of the outbreak was associated with a sick cow transported from an area in northeast China known to be affected with anthrax. A cow with a latent anthrax infection can begin its journey healthy but become ill en route or soon after arriving after lengthy trucking. Both the stress of travelling with little rest and water and the high summer temperatures will reduce the animal's innate resistance. Apart from the fact that an outbreak of cattle anthrax cases had occurred in the area in August 2011 [13], we found genetic proof that this sick cow in Ganyu had been infected with $B$. anthracis. Moreover, molecular typing showed that the genotype of the pagA gene amplified from the sick cow was identical to that from those four patients (patients 1-4 in Table 1) [14]. Our findings suggest that clinicians should consider $B$. anthracis as a potential agent of cutaneous infection in patients with a history of exposure to sick or dead livestock even in anthrax enzooticfree regions.

The outbreak of cutaneous anthrax that occurred in Ganyu County is the largest outbreak within Jiangsu Province to date. The attack rate of the outbreak was much higher than expected, which might be due to the fact that not all persons who had been exposed to the products of the sick cow were identified. According to the World Health Organization, only one human cutaneous case occurs for every 10 livestock carcasses in northern Europe [2]. In contrast, in Africa and Central Asia is frequently more than ten human cases per single infected carcass [2]. Previous studies have linked cutaneous $B$. anthracis outbreak with slaughtering of infected animals $[15,16]$. The outbreak occurred during summer when many of the persons exposed to the sick cow wore less clothing, hence increasing potential skin contact and making exposure more likely. Higher temperature and humidity also favour the formation of anthrax spores. In addition, along with cuts associated with manual slaughtering, both mosquito bites that are more common in summer and itches that are scratched by bloody fingernails all contribute to the presence of skin damage.

The occurrence of the outbreak indicates that it is critical for physicians, public health officials, and veterinarians to work together and share epidemic information to minimize the potential threat of anthrax. An effective surveillance system should be established to facilitate early detection, control and prevention of anthrax outbreaks. In order to prevent potential human infection, the quarantine of imported animals or their products from anthrax-endemic areas should be enhanced. The owners of livestock and butchers should also be educated regarding the need for personal protective equipment during the process of slaughtering and on health risks associated with slaughtering sick animals.

\section{ACKNOWLEDGEMENTS}

We thank the physicians who provided clinical information about cases, and Mike $\mathrm{He}$, from the Mailman School of Public Health, Columbia University for help in preparing the manuscript.

This work was supported in part by Jiangsu Province Health Development Project with Science and Education (no. ZX201109), Natural Science Foundation of China (no. 81373055), Jiangsu Province Science and Technology Support Program (no. BE2012769), and the 10th Summit of Six Top Talents of Jiangsu Province (no. 2013-WSN-061). The sponsors of the study had no role in the study design, data collection, data analysis, data interpretation, or writing of this report.

\section{SUPPLEMENTARY MATERIAL}

For supplementary material accompanying this paper visit http://dx.doi.org/10.1017/S0950268816001205 


\section{DECLARATION OF INTEREST}

None.

\section{REFERENCES}

1. Sweeney DA, et al. Anthrax infection. American Journal of Respiratory and Critical Care Medicine 2011; 184: 1333-1341.

2. OIE/WHO/FAO. Anthrax in Humans and Animals, 4th edn. Geneva: World Health Organization, 2008.

3. CDC. Human anthrax associated with an epizootic among livestock - North Dakota, 2000. Morbidity and Mortality Weekly Report 2001; 50: 677-680.

4. Cinquetti G, et al. Three related cases of cutaneous anthrax in France: clinical and laboratory aspects. Medicine (Baltimore) 2009; 88: 371-375.

5. CDC. Cutaneous anthrax associated with drum making using goat hides from West Africa - Connecticut, 2007. Morbidity and Mortality Weekly Report 2008; 57: 628-631.

6. Anaraki S, et al. Investigations and control measures following a case of inhalation anthrax in East London in a drum maker and drummer, October 2008. Eurosurveillance 2008; 13(51).

7. Grunow R, et al. Injection anthrax - a new outbreak in heroin users. Deutsches Arzteblatt International 2012; 109: 843-848.

8. Bartlett JG, Inglesby Jr. TV, Borio L. Management of anthrax. Clinical Infectious Diseases 2002; 35: 851-858.
9. Greene CM, et al. Epidemiologic investigations of bioterrorism-related anthrax, New Jersey, 2001. Emerging Infectious Diseases 2002; 8: 1048-1055.

10. Beyer W, Turnbull PC. Anthrax in animals. Molecular Aspects of Medicine 2009; 30: 481-489.

11. Kumar S, Tuteja $\mathbf{U}$. Detection of virulence-associated genes in clinical isolates of bacillus anthracis by multiplex PCR and DNA probes. Journal of Microbiology and Biotechnology 2009; 19: 1475-1481.

12. Woods CW, et al. Risk factors for human anthrax among contacts of anthrax-infected livestock in Kazakhstan. American Journal of Tropical Medicine and Hygiene 2004; 71: 48-52.

13. Yan MM, et al. Diagnosis and report of cattle anthrax outbreak in Liaoning Province [in Chinese]. Chinese Journal of Integrated Traditional and Western Medicine 2012; 4: 21-22.

14. Tan ZM, et al. Molecular characterization of Bacillus anthracis directly from patients' eschar and beef in an anthrax outbreak in Jiangsu Province, China, 2012. American Journal of Tropical Medicine and Hygiene 2014; 91: 574-576.

15. Reddy R, et al. Outbreak of cutaneous anthrax in Musalimadugu village, Chittoor district, Andhra Pradesh, India, July-August 2011. Journal of Infection in Developing Countries 2012; 6: 695-699.

16. Siddiqui MA, et al. Recent outbreak of cutaneous anthrax in Bangladesh: clinico-demographic profile and treatment outcome of cases attended at Rajshahi Medical College Hospital. BMC Research Notes 2012; 5: 464. 